\title{
Recovery of the Impulsive Diffusion Operator with Discontinuous Coefficient
}

\author{
Yaşar Çakmak ${ }^{1, *}$ and Seval Işı $k^{2}$ \\ ${ }^{1}$ Faculty Science, Department of Mathematics, Cumhuriyet University, 58140, Sivas, Turkey \\ ${ }^{2}$ Faculty of Education, Department of Secondary School Science and Mathematics Education, Cumhuriyet University, 58140, Sivas, \\ Turkey
}

Received: 24 Aug. 2013, Revised: 21 Nov. 2013, Accepted: 22 Nov. 2013

Published online: 1 Sep. 2014

\begin{abstract}
In this work impulsive diffusion operator with discontinuous coefficient is considered. Integral representation is derived and some important properties of eigenvalues are studied. Moreover, it is proven that the coefficients of the given problem are uniquely determined by the Weyl function.
\end{abstract}

Keywords: Diffusion equation, Discontinuous Coefficient, Discontinuity Conditions, Integral representation, Weyl function

\section{Introduction}

Inverse problems of spectral analysis consist in recovering operators from their spectral characteristics. Such problems often appear in applied mathematics, physics, and branches of natural sciences. Inverse problems also play important role in solving nonlinear evolution equations in mathematical physics. Physical applications of inverse problems can be found in the several works $[1-7]$.

First and most important results for inverse problem of a regular Sturm-Liouville operator were given by Ambarzumyan in 1929 [8] and Borg in 1945 [9]. In later years, inverse problems for regular Sturm-Liouville operator are solved in refs. $[10-15]$.

Inverse spectral problems for Sturm-Liouville operators with discontinuous coefficient appear in some physical problems. This kind of problems were investigated in several works $[16-20]$.

Generally, spectral problems for differential operators with the discontinuity conditions like

$$
\left\{\begin{array}{l}
y(d+0)=a y(d-0) \\
y^{\prime}(d+0)=b y^{\prime}(d-0)+c y(d-0)
\end{array}\right.
$$

were well-studied in refs. [21-24], where $a, b$ and $c$ are real numbers.
The diffusion equation or a quadratic pencil of SturmLiouville equation is written as

$$
-y^{\prime \prime}(x)+[2 \lambda p(x)+q(x)] y(x)=\lambda^{2} y(x), x \in[0, \pi]
$$

where $p(x) \in W_{2}^{m+1}[0, \pi], q(x) \in W_{2}^{m}[0, \pi] \quad(m \geq 0)$.

Direct and inverse problems for diffusion operators were studied by several authors in [25 - 37].

In this study, we consider a boundary value problem $L(p, q, d, \alpha, \beta, \gamma)$ of the form

$$
\ell y(x):=-y^{\prime \prime}(x)+[2 \lambda p(x)+q(x)] y(x)=\lambda^{2} \rho(x) y(x), x \in I
$$

with the boundary conditions

$$
U(y):=y^{\prime}(0, \lambda)=0, \quad V(y):=y(\pi, \lambda)=0
$$

and the discontinuity conditions

$$
\begin{aligned}
y(d+0) & =\beta y(d-0), \\
y^{\prime}(d+0) & =\beta^{-1} y^{\prime}(d-0)+\gamma y(d-0)
\end{aligned}
$$

where real valued functions

$$
p(x) \in W_{2}^{1}(0, \pi), q(x) \in L_{2}(0, \pi),
$$


$\lambda$ is a spectral parameter, $I=(0, d) \cup(d, \pi), \gamma$ and $\beta$ are real constants i.e., $|\beta-1|^{2}+\gamma^{2} \neq 0$ and

$$
\rho(x)=\left\{\begin{array}{c}
1, \quad 0 \leq x<d \\
\alpha^{2}, d<x \leq \pi
\end{array}, 0<\alpha<1 .\right.
$$

For the problem $L$, it is obtained a represantation for the solution and some important properties of eigenvalues are studied. Moreover, It is proven that the coefficients of the problem can be uniquely determined by Weyl function. The obtained results are generalizations of the similar results for the classical diffusion operator on a finite interval.

\section{Preliminaries}

In this section, we derive integral representation for the solution of equation (1) satisfying the conditions (2) and (3). Further some important properties of the spectrum of the problem $L$ will be learned.

Let the functions $S(x, \lambda), \varphi(x, \lambda), \psi(x, \lambda)$ be the solutions of the equation (1) satisfy the initial conditions

$$
\begin{aligned}
& S(0, \lambda)=0, S^{\prime}(0, \lambda)=1 \\
& \varphi(0, \lambda)=1, \varphi^{\prime}(0, \lambda)=0 \\
& \psi(\pi, \lambda)=0, \psi^{\prime}(\pi, \lambda)=1
\end{aligned}
$$

and the discontinuity conditions (3).

It is clear that the function $\psi(x, \lambda)$ satisfies the following integral equations

for $x>d$;

$\psi(x, \lambda)=\frac{\sin \lambda \alpha(x-\pi)}{\lambda \alpha}$

$+\frac{1}{\lambda \alpha} \int_{x}^{\pi} \sin \lambda \alpha(t-x)[2 \lambda p(t)+q(t)] \psi(t, \lambda) d t$,

for $x<d$;

$\psi(x, \lambda)=\frac{\beta^{+}}{\lambda} \sin \lambda\left(x-\mu^{+}(\pi)\right)+\frac{\beta}{\lambda}^{-} \sin \lambda\left(x-\mu^{-}(\pi)\right)$

$+\frac{\gamma}{2 \lambda^{2} \alpha}\left[\cos \lambda\left(x-\mu^{+}(\pi)\right)-\cos \lambda\left(x-\mu^{-}(\pi)\right)\right]$

$-\frac{1}{\lambda} \int_{d}^{\pi}\left[\beta^{+} \sin \lambda\left[x-\mu^{+}(t)\right]+\beta^{-} \sin \lambda\left[x-\mu^{-}(t)\right]\right] \times$

$\times[2 \lambda p(t)+q(t)] \psi(t, \lambda) d t$

$-\frac{1}{\lambda} \int_{x}^{d} \sin \lambda(x-t)[2 \lambda p(t)+q(t)] \psi(t, \lambda) d t$ $-\frac{\gamma}{2 \lambda^{2} \alpha} \int_{d}^{\pi}\left[\cos \lambda\left[x-\mu^{+}(t)\right]-\cos \lambda\left[x-\mu^{-}(t)\right]\right] \times$

$\times[2 \lambda p(t)+q(t)] \psi(t, \lambda) d t$,

where $\mu^{ \pm}(x)= \pm \alpha x \mp \alpha d+d$.

One can prove the following relations:

$$
\begin{gathered}
\psi(x, \lambda)= \begin{cases}O(\exp (|\tau|(\pi-x))), & x<d \\
O(\exp (|\tau| \alpha(\pi-x))), & x>d .\end{cases} \\
\psi^{\prime}(x, \lambda)= \begin{cases}O(|\lambda| \exp (|\tau|(\pi-x))), & x<d \\
O(|\lambda| \exp (|\tau| \alpha(\pi-x))), & x>d .\end{cases}
\end{gathered}
$$

uniformly in $x$ for $|\lambda| \rightarrow \infty$, where $\tau=\operatorname{Im} \lambda$.

Theorem 2.1. If $q(x) \in L_{2}(0, \pi), p(x) \in W_{2}^{1}(0, \pi)$, then there are the functions $A(x, t)$ and $B(x, t)$ whose first order partial derivatives are summable on $[0, \pi]$ for each $x \in[0, \pi]$ such that the representation

$$
\begin{aligned}
\varphi(x, \lambda)=\varphi_{0}(x, \lambda) & +\int_{0}^{\mu^{+}(x)} A(x, t) \cos \lambda t d t \\
& +\int_{0}^{\mu^{+}(x)} B(x, t) \sin \lambda t d t
\end{aligned}
$$

is provided, where $\beta^{ \pm}=\frac{1}{2}\left(\beta \pm \frac{1}{\alpha \beta}\right)$, $\mu^{ \pm}(x)= \pm \sqrt{\rho(x)} x+d(1 \mp \sqrt{\rho(x)}), \alpha(x)=\int_{0}^{x} p(t) d t$,

$$
\begin{array}{r}
\varphi_{0}(x, \lambda)=\beta^{+} \cos \left[\lambda \mu^{+}(x)-\frac{\alpha(x)}{\sqrt{\rho(x)}}\right] \\
+\beta^{-} \cos \left[\lambda \mu^{-}(x)+\frac{\alpha(x)}{\sqrt{\rho(x)}}\right] \\
\alpha(x)=x p(0) \\
+\frac{2 \rho(x)}{\beta^{+}} \int_{0}^{x}\left[A\left(\xi, \mu^{+}(\xi)\right) \sin \frac{\alpha(\xi)}{\sqrt{\rho(\xi)}}\right. \\
\left.-B\left(\xi, \mu^{+}(\xi)\right) \cos \frac{\alpha(\xi)}{\sqrt{\rho(\xi)}}\right] d \xi
\end{array}
$$

and in the following relations are satisfied :

$$
\begin{aligned}
& p(x)=p(0) \\
& +\frac{2 \rho(x)}{\beta^{-}}\left[A(x, t) \sin \frac{\alpha(x)}{\sqrt{\rho(x)}}\right. \\
& \left.+B(x, t) \cos \frac{\alpha(x)}{\sqrt{\rho(x)}}\right]\left.\right|_{t=\mu^{-}(x)-0} ^{\mu^{-}(x)+0}
\end{aligned}
$$




$$
\begin{aligned}
& q(x)=-\frac{p^{2}(x)}{\rho(x)} \\
& +\frac{2 \alpha}{\beta^{+}} \frac{d}{d x}\left[A\left(x, \mu^{+}(x)\right) \cos \frac{\alpha(x)}{\sqrt{\rho(x)}}\right. \\
& \left.+B\left(x, \mu^{+}(x)\right) \sin \frac{\alpha(x)}{\sqrt{\rho(x)}}\right] \\
& \beta^{-}\left[\frac{p^{2}(x)}{\rho(x)}+q(x)\right] \\
& =2 \alpha \frac{d}{d x}\left[A(x, t) \cos \frac{\alpha(x)}{\sqrt{\rho(x)}}\right. \\
& \left.-B(x, t) \sin \frac{\alpha(x)}{\sqrt{\rho(x)}}\right]\left.\right|_{t=\mu^{-}(x)-0} ^{\mu^{-}(x)+0} \\
& B(x, 0)=\left.A_{t}(x, t)\right|_{t=0}=0
\end{aligned}
$$

Moreover if we assume that $q(x) \in W_{2}^{1}(0, \pi), p(x) \in$ $W_{2}^{2}(0, \pi)$, the kernels $A(x, t)$ and $B(x, t)$ satisfy the system

$$
\left\{\begin{array}{l}
A_{x x}(x, t)-q(x) A(x, t)-2 p(x) B_{t}(x, t)=\rho(x) A_{t t}(x, t) \\
B_{x x}(x, t)-q(x) B(x, t)+2 p(x) A_{t}(x, t)=\rho(x) B_{t t}(x, t) .
\end{array}\right.
$$

If the second order derivatives of functions $A(x, t)$ and $B(x, t)$ are summable on $[0, \pi]$ for each $x \in[0, \pi]$ and these functions satisfy equalities (13) and relations (8) - (12), then $\varphi(x, \lambda)$ is a solution of equation (1) satifying initial conditions (2) and discontinuity conditions (3).

The function $\varphi(x, \lambda)$ is entire in $\lambda$ and the following asymptotic relations are valid for $|\lambda| \rightarrow \infty$.

$$
\text { If } x<d \text {, }
$$

$$
\begin{aligned}
\varphi(x, \lambda) & =O(\exp (|\tau| x)), \\
\varphi^{\prime}(x, \lambda) & =O(|\lambda| \exp (|\tau| x)) .
\end{aligned}
$$

If $x>d$,

$$
\begin{aligned}
\varphi(x, \lambda) & =O\left(\exp \left(|\tau| \mu^{+}(x)\right)\right), \\
\varphi^{\prime}(x, \lambda) & =O\left(|\lambda| \exp \left(|\tau| \mu^{+}(x)\right)\right) .
\end{aligned}
$$

Lemma 2.2. Let $\lambda_{n}$ and $\lambda_{k}\left(\lambda_{n} \neq \lambda_{k}\right)$ be the eigenvalues with the eigenfunctions $y\left(x, \lambda_{n}\right)$ and $y\left(x, \lambda_{k}\right)$, respectively. These functions are orthogonal in the sense of

$$
\begin{gathered}
\left(\lambda_{n}+\lambda_{k}\right) \int_{0}^{\pi} \rho(x) y\left(x, \lambda_{n}\right) y\left(x, \lambda_{k}\right) d x \\
-2 \int_{0}^{\pi} p(x) y\left(x, \lambda_{n}\right) y\left(x, \lambda_{k}\right) d x=0
\end{gathered}
$$

Proof.

Define $\ell y(x, \lambda)$ by

$$
\begin{aligned}
& \ell y(x, \lambda):=\frac{1}{\rho(x)}\left\{-y^{\prime \prime}(x, \lambda)+q(x) y(x, \lambda)\right\} \\
& =\lambda^{2} y(x, \lambda)-2 \lambda \frac{p(x)}{\rho(x)} y(x, \lambda),
\end{aligned}
$$

we get,

$$
\begin{aligned}
& \int_{0}^{\pi} \rho(x) \ell y\left(x, \lambda_{n}\right) y\left(x, \lambda_{k}\right) d x=\int_{0}^{\pi} \rho(x) y\left(x, \lambda_{n}\right) \ell y\left(x, \lambda_{k}\right) d x \\
& \int_{0}^{\pi} \rho(x)\left[\lambda_{n}^{2} y\left(x, \lambda_{n}\right)-2 \lambda_{n} \frac{p(x)}{\rho(x)} y\left(x, \lambda_{n}\right)\right] y\left(x, \lambda_{k}\right) d x \\
& =\int_{0}^{\pi} \rho(x) y\left(x, \lambda_{n}\right)\left[\lambda_{k}^{2} y\left(x, \lambda_{k}\right)-2 \lambda_{k} \frac{p(x)}{\rho(x)} y\left(x, \lambda_{k}\right)\right] d x .
\end{aligned}
$$

Thus

$$
\begin{aligned}
& \left(\lambda_{n}^{2}-\lambda_{k}^{2}\right) \int_{0}^{\pi} \rho(x) y\left(x, \lambda_{n}\right) y\left(x, \lambda_{k}\right) d x \\
& -2\left(\lambda_{n}-\lambda_{k}\right) \int_{0}^{\pi} p(x) y\left(x, \lambda_{n}\right) y\left(x, \lambda_{k}\right) d x=0 .
\end{aligned}
$$

By virtue of $\lambda_{n} \neq \lambda_{k}$,

$$
\begin{aligned}
& \left(\lambda_{n}+\lambda_{k}\right) \int_{0}^{\pi} \rho(x) y\left(x, \lambda_{n}\right) y\left(x, \lambda_{k}\right) d x \\
& -2 \int_{0}^{\pi} p(x) y\left(x, \lambda_{n}\right) y\left(x, \lambda_{k}\right) d x=0
\end{aligned}
$$

is obtained.

Let us define the function

$$
\Delta(\lambda)=W[\psi, \varphi]=\psi(x, \lambda) \varphi^{\prime}(x, \lambda)-\psi^{\prime}(x, \lambda) \varphi(x, \lambda)
$$

Since $\Delta(\lambda)$ is constant on $x \in[0, d) \cup(d, \pi]$, we get

$$
\Delta(\lambda)=-\psi^{\prime}(0, \lambda)=-\varphi(\pi, \lambda) .
$$

$\Delta(\lambda)$ is called the characteristic function of the problem $L$. The function $\Delta(\lambda)$ is entire in $\lambda$ and it has at most a countable set of zeros $\left\{\lambda_{n}\right\}$.

Lemma 2.3. The zeros $\left\{\lambda_{n}\right\}$ of the characteristic function coincide with the eigenvalues of the boundary value problem $L$ and for eigenfunctions $\varphi\left(x, \lambda_{n}\right)$ and $\psi\left(x, \lambda_{n}\right)$ there exists a sequence $\left\{\beta_{n}\right\}$, such that the relations

$$
\psi\left(x, \lambda_{n}\right)=\beta_{n} \varphi\left(x, \lambda_{n}\right)
$$


holds.

Proof.

Let $\lambda_{0}$ be a zero of the function $\Delta(\lambda)$. Then $\Delta\left(\lambda_{0}\right)=$ 0 . So, we get $\psi\left(x, \lambda_{0}\right)=\beta_{0} \varphi\left(x, \lambda_{0}\right)$ for some constant $\beta_{0}$. Hence $\lambda_{0}$ is an eigenvalue and $\psi\left(x, \lambda_{0}\right)$ and $\varphi\left(x, \lambda_{0}\right)$ are eigenfunctions related to $\lambda_{0}$.

Let $\lambda_{0}$ be an eigenvalue of the problem $L$ and show that $\Delta\left(\lambda_{0}\right)=0$. Suppose that $\Delta\left(\lambda_{0}\right) \neq 0$. Then the functions $\varphi\left(x, \lambda_{0}\right)$ and $\psi\left(x, \lambda_{0}\right)$ are linearly independent. Thus

$$
y\left(x, \lambda_{0}\right)=C_{1} \psi\left(x, \lambda_{0}\right)+C_{2} \varphi\left(x, \lambda_{0}\right)
$$

is a general solution of the problem $L$. Hence

$$
\psi\left(x, \lambda_{0}\right)=\frac{1}{C_{1}} y\left(x, \lambda_{0}\right)-\frac{C_{2}}{C_{1}} \varphi\left(x, \lambda_{0}\right),
$$

where $C_{1} \neq 0$.

The equality

$$
<\psi\left(x, \lambda_{0}\right), \varphi\left(x, \lambda_{0}\right)>=0
$$

contradicts with $\Delta\left(\lambda_{0}\right) \neq 0$.

Since function $\varphi\left(x, \lambda_{n}\right)$ and $\psi\left(x, \lambda_{n}\right)$ are linearly dependent, there exists sequence $\beta_{n}$ such that

$$
\psi\left(x, \lambda_{n}\right)=\beta_{n} \varphi\left(x, \lambda_{n}\right) .
$$

Let

$$
\begin{aligned}
\Delta_{0}(\lambda) & =\beta^{+} \cos \left[\lambda \mu^{+}(\pi)-\frac{\alpha(\pi)}{\alpha}\right] \\
& +\beta^{-} \cos \left[\lambda \mu^{-}(\pi)+\frac{\alpha(\pi)}{\alpha}\right]
\end{aligned}
$$

and $\left\{\lambda_{n}^{0}\right\}$ are zeros of $\Delta_{0}(\lambda)$.

From [36], the roots of the equation $\Delta_{0}\left(\lambda_{n}^{0}\right)=0$ have the form

$$
\lambda_{n}^{0}=\frac{n \pi}{\mu^{+}(\pi)}+\frac{\alpha(\pi)}{\alpha \mu^{+}(\pi)}+h_{n}, \sup _{n}\left|h_{n}\right|<+\infty .
$$

Moreover, denote

$$
\alpha_{n}:=\int_{0}^{\pi} \rho(x) y^{2}\left(x, \lambda_{n}\right) d x-\frac{1}{\lambda_{n}} \int_{0}^{\pi} p(x) y^{2}\left(x, \lambda_{n}\right) d x
$$

The numbers $\left\{\alpha_{n}\right\}$ are called normalizing numbers of problem $L$.

Lemma 2.4. Eigenvalues of the problem $L$ are simple, i.e., $\dot{\Delta}\left(\lambda_{n}\right) \neq 0$ and the eigenvalues of the boundary value problem $L$ are real. $\left(\dot{\Delta}(\lambda)=\frac{d \Delta(\lambda)}{d \lambda}\right)$

\section{Proof.}

Since $\varphi=\varphi(x, \lambda)$ and $\psi=\psi(x, \lambda)$ are the solutions of the equation (1), the equalities

$$
\begin{aligned}
-\varphi^{\prime \prime}+[2 \lambda p(x)+q(x)] \varphi & =\lambda^{2} \rho(x) \varphi \\
-\dot{\varphi}^{\prime \prime}+[2 \lambda p(x)+q(x)] \dot{\varphi} & =\lambda^{2} \rho(x) \dot{\varphi}+\{2 \lambda \rho(x)-2 p(x)\} \varphi \\
-\psi^{\prime \prime}+[2 \lambda p(x)+q(x)] \psi & =\lambda^{2} \rho(x) \psi \\
-\dot{\psi}^{\prime \prime}+[2 \lambda p(x)+q(x)] \dot{\psi} & =\lambda^{2} \rho(x) \dot{\psi}+\{2 \lambda \rho(x)-2 p(x)\} \psi
\end{aligned}
$$

hold.

If the first equation is multiplied by $\dot{\psi}$, the last equation is multiplied by $\varphi$ and substracting them side by side, and if the second equation is multiplied by $\psi$, the third equation is multiplied by $\dot{\varphi}$ and substracting them side by side the equalities

$$
\begin{aligned}
& \frac{d}{d x}\left\{\varphi \dot{\psi}^{\prime}-\varphi^{\prime} \dot{\psi}\right\}=-\{2 \lambda \rho(x)-2 p(x)\} \varphi \psi \\
& \frac{d}{d x}\left\{\dot{\varphi} \psi^{\prime}-\dot{\varphi}^{\prime} \psi\right\}=\{2 \lambda \rho(x)-2 p(x)\} \varphi \psi
\end{aligned}
$$

are obtained. Finally, integrating above equations over $[x, \pi],[0, x]$, respectively, we get

$$
\begin{aligned}
& -\left.\left\{\varphi(\xi, \lambda) \dot{\psi}^{\prime}(\xi, \lambda)-\varphi^{\prime}(\xi, \lambda) \dot{\psi}(\xi, \lambda)\right\}\right|_{x} ^{\pi} \\
& =\int_{x}^{\pi}[2 \lambda \rho(\xi)-2 p(\xi)] \varphi(\xi, \lambda) \psi(\xi, \lambda) d \xi
\end{aligned}
$$

and

$$
\begin{aligned}
& \left.\left\{\dot{\varphi}(\xi, \lambda) \psi^{\prime}(\xi, \lambda)-\dot{\varphi}^{\prime}(\xi, \lambda) \psi(\xi, \lambda)\right\}\right|_{0} ^{x} \\
& =\int_{0}^{x}[2 \lambda \rho(\xi)-2 p(\xi)] \varphi(\xi, \lambda) \psi(\xi, \lambda) d \xi
\end{aligned}
$$

If the last equations are added them side by side, the equality

$$
\begin{aligned}
& W[\varphi, \dot{\psi}]+W[\dot{\varphi}, \psi]=-\dot{\Delta}(\lambda) \\
& =\int_{0}^{\pi}[2 \lambda \rho(\xi)-2 p(\xi)] \varphi(\xi, \lambda) \psi(\xi, \lambda) d \xi
\end{aligned}
$$

is obtained. Hence,

$$
\dot{\Delta}(\lambda)=-\int_{0}^{\pi}[2 \lambda \rho(\xi)-2 p(\xi)] \varphi(\xi, \lambda) \psi(\xi, \lambda) d \xi
$$

Writing $\lambda=\lambda_{n}$ and by virtue of (21), we get 
$\dot{\Delta}\left(\lambda_{n}\right)=-\int_{0}^{\pi}\left[2 \lambda_{n} \rho(\xi)-2 p(\xi)\right] \beta_{n} \varphi^{2}\left(\xi, \lambda_{n}\right) d \xi$

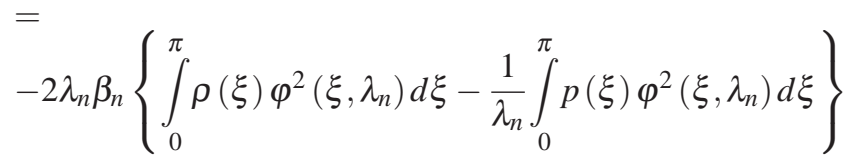

$=-2 \lambda_{n} \beta_{n} \alpha_{n}$

It is obviously that $\dot{\Delta}\left(\lambda_{n}\right) \neq 0$ for $\lambda_{n} \neq 0$.

On the other hand, the conditions

$$
y^{\prime}(0) \overline{y(0)}-y^{\prime}(\pi) \overline{y(\pi)}=0
$$

and

$$
\int_{0}^{\pi}\left\{\left|y^{\prime}(x)\right|^{2}+q(x)|y(x)|^{2}\right\} d x>0
$$

imply that the eigenvalues of the boundary value problem $L$ are real, where $y(x) \neq 0$ and $y(x) \in W_{2}^{2}([0, d) \cup(d, \pi])$.

Theorem 2.5. Eigenvalues of the problem $L$ have the asymptotic behaviour

$$
\lambda_{n}=\lambda_{n}^{0}+\frac{d_{n}}{\lambda_{n}^{0}}+\frac{k_{n}}{\lambda_{n}^{0}}, n \rightarrow \infty
$$

where $d_{n}$ is a bounded sequence, $k_{n} \in l_{2}$,

$$
\lambda_{n}^{0}=\frac{n \pi}{\mu^{+}(\pi)}+\frac{\alpha(\pi)}{\alpha \mu^{+}(\pi)}+h_{n}, \sup _{n}\left|h_{n}\right|<+\infty .
$$

\section{Proof.}

Let us denote $G_{\delta}=\left\{\lambda:\left|\lambda-\lambda_{n}^{0}\right| \geq \delta\right\}$ and $\Gamma_{n}=\left\{\lambda:|\lambda|=\left|\lambda_{n}^{0}\right|+\frac{\beta}{2}\right\}$, where $\delta$ is a sufficiently small positive number $\delta<\frac{\beta}{2}$. Using Theorem 12.4 [41], it is easy to show that

$$
\left|\Delta_{0}(\lambda)\right| \geq C_{\delta} e^{|\operatorname{Im} \lambda| \mu^{+}(\pi)}>\left|\Delta(\lambda)-\Delta_{0}(\lambda)\right|
$$

for $\lambda \in \Gamma_{n}$.

On the other hand, by virtue of lemma 1.3.1.in [42] and from [39], we obtain

$$
\Delta(\lambda)-\Delta_{0}(\lambda)=O\left(e^{|\operatorname{Im} \lambda| \mu^{+}(\pi)}\right),|\lambda| \rightarrow \infty .
$$

Then by Rouché theorem, number of zeros of the function $\left\{\Delta(\lambda)-\Delta_{0}(\lambda)\right\}+\Delta_{0}(\lambda)=\Delta(\lambda)$ inside the contour $\Gamma_{n}$ coincides with the number of zeros of function $\Delta_{0}(\lambda)$. Further, applying the Rouché theorem to the circle $\gamma_{n}(\delta)=\left\{\lambda:\left|\lambda-\lambda_{n}^{0}\right| \leq \delta\right\}$, we conclude that for sufficiently large $n$, there exists only one zero $\lambda_{n}$ of the function $\Delta(\lambda)$ in $\gamma_{n}(\delta)$. By virtue of the arbitrariness of $\delta>0$, we have

$$
\lambda_{n}=\lambda_{n}^{0}+\varepsilon_{n}, \varepsilon_{n}=o(1), n \rightarrow \infty .
$$

By virtue of $\Delta\left(\lambda_{n}\right)=0$, we get

$$
\begin{array}{r}
\Delta_{0}\left(\lambda_{n}^{0}+\varepsilon_{n}\right)+\int_{0}^{\mu^{+}(\pi)} A(\pi, t) \cos \left(\lambda_{n}^{0}+\varepsilon_{n}\right) t d t \\
+\int_{0}^{\mu^{+}(\pi)} B(\pi, t) \sin \left(\lambda_{n}^{0}+\varepsilon_{n}\right) t d t=0
\end{array}
$$

From (19),

$$
\Delta_{0}\left(\lambda_{n}^{0}+\varepsilon_{n}\right)=\left[\dot{\Delta}_{0}\left(\lambda_{n}^{0}\right)+o(1)\right] \varepsilon_{n}, n \rightarrow \infty .
$$

Thus (24) can be rewritten as form of

$$
\begin{aligned}
& \dot{\Delta}_{0}\left(\lambda_{n}^{0}\right) \varepsilon_{n}+\int_{0}^{\mu^{+}(\pi)} A(\pi, t) \cos \left(\lambda_{n}^{0}+\varepsilon_{n}\right) t d t \\
& +\int_{0}^{\mu^{+}(\pi)} B(\pi, t) \sin \left(\lambda_{n}^{0}+\varepsilon_{n}\right) t d t+o\left(\varepsilon_{n}\right), n \rightarrow \infty .
\end{aligned}
$$

Further we know that since $\Delta_{0}(\lambda)$ is type of "Sine type" $[40]$, there exists $\gamma_{\delta}>0$ such that $\left|\dot{\Delta}_{0}\left(\lambda_{n}^{0}\right)\right| \geq \gamma_{\delta}>0$ is satisfied for all $n$.

Substituting (20) into (25), we obtained

$$
\begin{aligned}
& \varepsilon_{n} \approx \frac{1}{2 \lambda_{n}^{0} \dot{\Delta}_{0}\left(\lambda_{n}^{0}\right)}\left\{\left[\frac{\beta^{-}}{\alpha} \sin \left[\frac{\alpha(\pi)}{\alpha}+\left(\lambda_{n}^{0}+\varepsilon_{n}\right) \mu^{-}(\pi)\right]\right.\right. \\
& \left.+\frac{\beta^{+}}{\alpha} \sin \left[\frac{\alpha(\pi)}{\alpha}-\left(\lambda_{n}^{0}+\varepsilon_{n}\right) \mu^{+}(\pi)\right]\right] \int_{0}^{\pi}\left[q(t)+\left(\frac{p(t)}{\alpha}\right)^{2}\right] d t \\
& -\left[\frac{\beta^{-}}{\alpha^{2}} \cos \left[\frac{\alpha(\pi)}{\alpha}-\left(\lambda_{n}^{0}+\varepsilon_{n}\right) \mu^{-}(\pi)\right]\right. \\
& \left.\left.+\frac{\beta^{+}}{\alpha} \cos \left[\frac{\alpha(\pi)}{\alpha}-\left(\lambda_{n}^{0}+\varepsilon_{n}\right) \mu^{+}(\pi)\right]\right][p(\pi)-p(0)]\right\}
\end{aligned}
$$$$
+\frac{1}{\lambda_{n}^{0} \dot{\Delta}_{0}\left(\lambda_{n}^{0}\right)}\left[\int_{0}^{\mu^{+}(\pi)} A_{t}(\pi, t) \sin \left(\lambda_{n}^{0}+\varepsilon_{n}\right) t d t\right.
$$$$
\left.-\int_{0}^{\mu^{+}(\pi)} B_{t}(\pi, t) \cos \left(\lambda_{n}^{0}+\varepsilon_{n}\right) t d t\right]
$$

Since for $\varepsilon_{n} \rightarrow 0, \quad \cos \varepsilon_{n} \mu^{ \pm}(\pi) \sim 1$, $\sin \varepsilon_{n} \mu^{ \pm}(\pi) \sim \varepsilon_{n} \mu^{ \pm}(\pi)$, we obtain 


$$
\begin{aligned}
& \varepsilon_{n} \approx \frac{1}{2 \lambda_{n}^{0} \dot{\Delta}_{0}\left(\lambda_{n}^{0}\right)}\left\{\left[\frac{\beta^{-}}{\alpha} \sin \left[\lambda_{n}^{0} \mu^{-}(\pi)+\frac{\alpha(\pi)}{\alpha}\right]\right.\right. \\
& \left.-\frac{\beta^{+}}{\alpha} \sin \left[\lambda_{n}^{0} \mu^{+}(\pi)-\frac{\alpha(\pi)}{\alpha}\right]\right] \int_{0}^{\pi}\left[q(t)+\left(\frac{p(t)}{\alpha}\right)^{2}\right] d t \\
& -\left[\frac{\beta^{-}}{\alpha^{2}} \cos \left[\lambda_{n}^{0} \mu^{-}(\pi)+\frac{\alpha(\pi)}{\alpha}\right]\right. \\
& \left.\left.+\frac{\beta^{+}}{\alpha} \cos \left[\lambda_{n}^{0} \mu^{+}(\pi)-\frac{\alpha(\pi)}{\alpha}\right]\right][p(\pi)-p(0)]\right\} \\
& +\frac{1}{\lambda_{n}^{0} \dot{\Delta}_{0}\left(\lambda_{n}^{0}\right)}\left[\int_{0}^{\mu^{+}(\pi)} A_{t}(\pi, t) \sin \lambda_{n}^{0} t d t-\int_{0}^{\mu^{+}(\pi)} B_{t}(\pi, t) \cos \lambda_{n}^{0} t d t\right] \\
& +\frac{o\left(\varepsilon_{n}\right)}{\lambda_{n}^{0}}, n \rightarrow \infty .
\end{aligned}
$$

Moreover by the virtue of

$$
\left\{\int_{0}^{\mu^{+}(\pi)} A_{t}(\pi, t) \sin \left(\lambda_{n}^{0}+\varepsilon_{n}\right) t d t\right\} \in l_{2}
$$

and

$$
\left\{\int_{0}^{\mu^{+}(\pi)} B_{t}(\pi, t) \cos \left(\lambda_{n}^{0}+\varepsilon_{n}\right) t d t\right\} \in l_{2}
$$

we get

$$
\begin{aligned}
& \varepsilon_{n} \approx \frac{1}{2 \lambda_{n}^{0} \dot{\Delta}_{0}\left(\lambda_{n}^{0}\right)}\left\{\left[\frac{\beta^{-}}{\alpha} \sin \left[\lambda_{n}^{0} \mu^{-}(\pi)+\frac{\alpha(\pi)}{\alpha}\right]\right.\right. \\
& \left.-\frac{\beta^{+}}{\alpha} \sin \left[\lambda_{n}^{0} \mu^{+}(\pi)-\frac{\alpha(\pi)}{\alpha}\right]\right] \int_{0}^{\pi}\left[q(t)+\left(\frac{p(t)}{\alpha}\right)^{2}\right] d t \\
& -\left[\frac{\beta^{-}}{\alpha^{2}} \cos \left[\lambda_{n}^{0} \mu^{-}(\pi)+\frac{\alpha(\pi)}{\alpha}\right]\right. \\
& \left.\left.+\frac{\beta^{+}}{\alpha} \cos \left[\lambda_{n}^{0} \mu^{+}(\pi)-\frac{\alpha(\pi)}{\alpha}\right]\right][p(\pi)-p(0)]\right\} \\
& +\frac{k_{n}}{\lambda_{n}^{0}}
\end{aligned}
$$

where $k_{n} \in l_{2}$.

Hence

$$
\lambda_{n}=\lambda_{n}^{0}+\frac{d_{n}}{\lambda_{n}^{0}}+\frac{k_{n}}{\lambda_{n}^{0}}, n \rightarrow \infty
$$

where

$d_{n}=\frac{1}{2 \dot{\Delta}_{0}\left(\lambda_{n}^{0}\right)}\left\{\left[\frac{\beta^{-}}{\alpha} \sin \left[\lambda_{n}^{0} \mu^{-}(\pi)+\frac{\alpha(\pi)}{\alpha}\right]\right.\right.$

$$
\begin{aligned}
& \left.-\frac{\beta^{+}}{\alpha} \sin \left[\lambda_{n}^{0} \mu^{+}(\pi)-\frac{\alpha(\pi)}{\alpha}\right]\right] \int_{0}^{\pi}\left[q(t)+\left(\frac{p(t)}{\alpha}\right)^{2}\right] d t \\
& -\left[\frac{\beta^{-}}{\alpha^{2}} \cos \left[\lambda_{n}^{0} \mu^{-}(\pi)+\frac{\alpha(\pi)}{\alpha}\right]\right. \\
& \left.\left.+\frac{\beta^{+}}{\alpha} \cos \left[\lambda_{n}^{0} \mu^{+}(\pi)-\frac{\alpha(\pi)}{\alpha}\right]\right][p(\pi)-p(0)]\right\}
\end{aligned}
$$

is a bounded sequence.

Consequently, theorem is proved.

\section{Main Result}

In this section the uniqueness theorem of inverse problem according to Weyl function is proved.

We consider a second problem $\widetilde{L}$

$$
\left\{\begin{array}{l}
\tilde{\ell} y(x)=-y^{\prime \prime}(x)+[2 \lambda p(x)+\widetilde{q}(x)] y(x)=\lambda^{2} \rho(x) y(x), x \in \widetilde{I} \\
y^{\prime}(0, \lambda)=0, \quad y(\pi, \lambda)=0 \\
y(\widetilde{d}+0)=\widetilde{\beta} y(\widetilde{d}-0) \\
y^{\prime}(\widetilde{d}+0)=\widetilde{\beta}^{-1} y^{\prime}(\widetilde{d}-0)+\widetilde{\gamma} y(\widetilde{d}-0)
\end{array}\right.
$$

where $\widetilde{q}(x)$ has the same properties of $q(x)$ and $\widetilde{I}=(0, \widetilde{d}) \cup(\widetilde{d}, \pi)$. We agree that if a certain symbol $\delta$ denotes an object related to $L$, then $\widetilde{\delta}$ will denote an analogous object related to $\widetilde{L}$.

Let $\Phi(x, \lambda)$ be the solution of (1) under the conditions $U(\Phi)=1, V(\Phi)=0$ and under the jump conditions. Denote

$\Phi(x, \lambda):=-\frac{\psi(x, \lambda)}{\Delta(\lambda)}$ and $M(\lambda):=\Phi(0, \lambda)=-\frac{\psi(0, \lambda)}{\Delta(\lambda)}$.

The functions $\Phi(x, \lambda)$ and $M(\lambda)$ are called the Weyl solution and the Weyl function [38] of the boundary value problem $L$, respectively. It is clear that $M(\lambda)$ is a meromorphic function with poles in $\left\{\lambda_{n}\right\}_{n>0}$.

Lemma 3.1. The following equation is valid except for the eigenvalues $\left\{\lambda_{n}\right\}_{n \geq 0}$ :

$$
\Phi(x, \lambda)=S(x, \lambda)+M(\lambda) \varphi(x, \lambda)
$$

Proof.

One can prove in a direct calculation that

$$
\left.W[\varphi, S]\right|_{x=0}=1 \neq 0 .
$$

So the functions $\varphi(x, \lambda)$ and $S(x, \lambda)$ are linearly independent. Thus, the function $\psi(x, \lambda)$ can be written as follows:

$$
\psi(x, \lambda)=A(\lambda) \varphi(x, \lambda)+B(\lambda) S(x, \lambda)
$$


From the initial conditions (4), we calculate

$A(\lambda)=\psi(0, \lambda)$ and $B(\lambda)=\psi^{\prime}(0, \lambda)=-\Delta(\lambda)$.

This completes the proof.

Theorem 3.2. If $M(\lambda)=\widetilde{M}(\lambda)$, then $L=\widetilde{L}$. Thus, given the Weyl function uniquely determines the boundary value problem $L$.

Proof:

Let us define the matrix $P(x, \lambda)=\left[P_{j, k}(x, \lambda)\right]$, $(j, k=1,2)$ by the formula

$$
P(x, \lambda)\left[\begin{array}{cc}
\widetilde{\varphi}(x, \lambda) & \widetilde{\Phi}(x, \lambda) \\
\widetilde{\varphi}^{\prime}(x, \lambda) & \widetilde{\Phi}^{\prime}(x, \lambda)
\end{array}\right]=\left[\begin{array}{cc}
\varphi(x, \lambda) & \Phi(x, \lambda) \\
\varphi^{\prime}(x, \lambda) & \Phi^{\prime}(x, \lambda)
\end{array}\right]
$$

Rewrite (29) in the form

$$
\left\{\begin{array}{l}
P_{11}(x, \lambda)=\varphi(x, \lambda) \widetilde{\Phi}^{\prime}(x, \lambda)-\widetilde{\varphi}^{\prime}(x, \lambda) \Phi(x, \lambda) \\
P_{12}(x, \lambda)=\widetilde{\varphi}(x, \lambda) \Phi(x, \lambda)-\varphi(x, \lambda) \widetilde{\Phi}(x, \lambda) \\
P_{21}(x, \lambda)=\varphi^{\prime}(x, \lambda) \widetilde{\Phi}^{\prime}(x, \lambda)-\widetilde{\varphi}^{\prime}(x, \lambda) \Phi^{\prime}(x, \lambda) \\
P_{22}(x, \lambda)=\widetilde{\varphi}(x, \lambda) \Phi^{\prime}(x, \lambda)-\varphi^{\prime}(x, \lambda) \widetilde{\Phi}(x, \lambda)
\end{array}\right.
$$

or

$$
\left\{\begin{array}{l}
P_{11}(x, \lambda)=\varphi(x, \lambda) \frac{\widetilde{\psi}^{\prime}(x, \lambda)}{\widetilde{\Delta}(\lambda)}-\widetilde{\varphi}^{\prime}(x, \lambda) \frac{\psi(x, \lambda)}{\Delta(\lambda)} \\
P_{12}(x, \lambda)=\widetilde{\varphi}(x, \lambda) \frac{\psi(x, \lambda)}{\Delta(\lambda)}-\varphi(x, \lambda) \frac{\widetilde{\psi}(x, \lambda)}{\widetilde{\Delta}(\lambda)} \\
P_{21}(x, \lambda)=\varphi^{\prime}(x, \lambda) \frac{\widetilde{\psi}^{\prime}(x, \lambda)}{\widetilde{\Delta}(\lambda)}-\widetilde{\varphi}^{\prime}(x, \lambda) \frac{\psi^{\prime}(x, \lambda)}{\Delta(\lambda)} \\
P_{22}(x, \lambda)=\widetilde{\varphi}(x, \lambda) \frac{\psi^{\prime}(x, \lambda)}{\Delta(\lambda)}-\varphi^{\prime}(x, \lambda) \frac{\widetilde{\psi}(x, \lambda)}{\widetilde{\Delta}(\lambda)}
\end{array}\right.
$$

It follows from (3)

$$
\begin{aligned}
& P_{11}(x, \lambda)=\varphi(x, \lambda) \widetilde{S}^{\prime}(x, \lambda)-\widetilde{\varphi}^{\prime}(x, \lambda) S(x, \lambda) \\
& +[\widetilde{M}(\lambda)-M(\lambda)] \varphi(x, \lambda) \widetilde{\varphi}^{\prime}(x, \lambda) \\
& P_{12}(x, \lambda)=\widetilde{\varphi}(x, \lambda) S(x, \lambda)-\varphi(x, \lambda) \widetilde{S}(x, \lambda) \\
& +[M(\lambda)-\widetilde{M}(\lambda)] \varphi(x, \lambda) \widetilde{\varphi}(x, \lambda) \\
& P_{21}(x, \lambda)=\varphi^{\prime}(x, \lambda) \widetilde{S}^{\prime}(x, \lambda)-\widetilde{\varphi}^{\prime}(x, \lambda) S^{\prime}(x, \lambda) \\
& +[\widetilde{M}(\lambda)-M(\lambda)] \varphi^{\prime}(x, \lambda) \widetilde{\varphi}^{\prime}(x, \lambda) \\
& P_{22}(x, \lambda)=\widetilde{\varphi}(x, \lambda) S^{\prime}(x, \lambda)-\varphi^{\prime}(x, \lambda) \widetilde{S}(x, \lambda) \\
& +[M(\lambda)-\widetilde{M}(\lambda)] \varphi^{\prime}(x, \lambda) \widetilde{\varphi}(x, \lambda) .
\end{aligned}
$$

Thus if $M(\lambda) \equiv \widetilde{M}(\lambda)$ then the functions $P_{j, k}(x, \lambda)$, $(j, k=1,2) \quad$ are entire in $\lambda$. Denote $\Lambda_{\delta}:=\left\{\lambda:\left|\lambda-\lambda_{n}\right|>\delta\right\}$, where $\delta$ is sufficiently small number. Taking $(5)-(6)$ and (14) - (15) into account we get

$$
\left|P_{11}(x, \lambda)\right| \leq C_{\delta} \text { and }\left|P_{12}(x, \lambda)\right| \leq \frac{C_{\delta}}{|\lambda|}, x \in[0, \pi]
$$

for $\lambda$ in $\Lambda_{\delta} \cap \widetilde{\Lambda}_{\delta}$.

According to the last inequalities and Liouville's theorem, we obtain for $\forall x \in[0, \pi] \backslash\{d, \widetilde{d}\}$

$$
P_{11}(x, \lambda)=A(x) \text { and } P_{12}(x, \lambda)=0 .
$$

It follows from (30) and (32)

$$
\varphi(x, \lambda) \widetilde{\Phi}^{\prime}(x, \lambda)-\widetilde{\varphi}^{\prime}(x, \lambda) \Phi(x, \lambda)=A(x)
$$

and

$$
\widetilde{\varphi}(x, \lambda) \Phi(x, \lambda)-\varphi(x, \lambda) \widetilde{\Phi}(x, \lambda)=0 .
$$

Multiplying $(33)$ by $\widetilde{\varphi}(x, \lambda),(34)$ by $\widetilde{\varphi}^{\prime}(x, \lambda)$ and subtracting them by side, we obtain

$$
\begin{gathered}
\widetilde{\varphi}(x, \lambda) A(x)=\varphi(x, \lambda)\left[\widetilde{\varphi}(x, \lambda) \widetilde{\Phi}^{\prime}(x, \lambda)-\widetilde{\varphi}^{\prime}(x, \lambda) \widetilde{\Phi}(x, \lambda)\right] . \\
\text { Since } W[\widetilde{\varphi}, \widetilde{\Phi}]=1, \\
\varphi(x, \lambda)=\widetilde{\varphi}(x, \lambda) A(x) .
\end{gathered}
$$

Similarly since $W[\varphi, \Phi]=1$,

$$
\Phi(x, \lambda)=\widetilde{\Phi}(x, \lambda) A(x) .
$$

By virtue of (35) and (36) we get $A^{2}(x) \equiv 1$, for all $x$ in $[0, \pi] \backslash\{d, \widetilde{d}\}$ and for all $\lambda$.

On the other hand, the asymptotic expressions

$$
\begin{aligned}
& \varphi(x, \lambda)=C \exp \left(-i\left(\lambda \mu^{+}(x)-\frac{\alpha(x)}{\sqrt{\rho(x)}}\right)\right)\left(1+O\left(\frac{1}{\lambda}\right)\right) \\
& \widetilde{\varphi}(x, \lambda)=\widetilde{C} \exp \left(-i\left(\lambda \mu^{+}(x)-\frac{\alpha(x)}{\sqrt{\rho(x)}}\right)\right)\left(1+O\left(\frac{1}{\lambda}\right)\right)
\end{aligned}
$$

are valid for sufficiently large $\lambda$ on the imaginary axis, where

$$
C=\left\{\begin{array}{l}
\frac{1}{2}, \text { if } x<d \\
\frac{\beta^{+}}{2}, \text { if } x>d
\end{array} \text { and } \widetilde{C}=\left\{\begin{array}{l}
\frac{1}{2}, \text { if } x<\widetilde{d} \\
\frac{\widetilde{\beta}^{+}}{2}, \text { if } x>\widetilde{d}
\end{array}\right.\right.
$$


Firstly, let us show that $d=\widetilde{d}$. Assume in contrary that $d \neq \widetilde{d}$. Without loss of generality we assume $d<\widetilde{d}$. From (35), (37) and (38) we have

$$
\left\{\begin{array}{l}
A(x)=1, \quad \text { for } x \in[0, d) \\
A(x)=\beta^{+}, \text {for } x \in(d, \widetilde{d}) \\
A(x)=\frac{\beta^{+}}{\widetilde{\beta}^{+}}, \text {for } x \in(\widetilde{d}, \pi] .
\end{array}\right.
$$

Since $A^{2}(x) \equiv 1$ and $\beta^{+} \neq 1$, we obtain $d=\widetilde{d}, A(x) \equiv$ 1 and $\beta^{+}=\widetilde{\beta}^{+}$.Thus

$\varphi(x, \lambda)=\widetilde{\varphi}(x, \lambda)$ and $\Phi(x, \lambda)=\widetilde{\Phi}(x, \lambda)$ for all $x$ and $\lambda$.

Hence $q(x)=\widetilde{q}(x)$ a.e. in $(0, \pi), \beta=\widetilde{\beta}$ and $\gamma=\widetilde{\gamma}$. Consequently, $L=\widetilde{L}$.

\section{References}

[1] M. Kac, Can one hear the shape of a drum, The American Mathematical Monthly, 73, 1-23 (1966).

[2] A. V. Likov and Yu. A. Mikhailov, The theory of heat and mass transfer, Gosnergoizdat, (1963). [in Russian]

[3] O. N. Litvinenko, V. I. Soshnikov, The theory of heteregeneous lines and their applications in radio engineering, Radio, Moscow, (1964). [in Russian]

[4] J. McLaughlin and P. Polyakov, On the uniqueness of a spherical symmetric speed of sound from transmission eigenvalues, J. Diff. Eqn., 107, 351-382 (1994).

[5] V. P. Meschanov and A. L. Feldstein, Automatic design of directional couplers, Sviaz, Moscow, (1980).

[6] A. N. Tikhonov and A. A. Samarskii, Equations of mathematical physics, Pergamon, Oxford, (1990).

[7] N. N. Voitovich, B. Z. Katsenelbaum, and A. N. Sivov, Generalized method of eigen-vibration in the theory of diffraction [M], Nauka, Moskov, (1997). [in Russian]

[8] V. A. Ambartsumyan, Über eine frage der eigenwerttheorie, Z. Physik, 53, 690-695 (1929).

[9] G. Borg, Eine umkehrung der Sturm-Liouvilleschen eigenwertaufgabe bestimmung der differentialgleichung durch die eigenwerte, Acta Math., 78, 1-96 (1946).

[10] I. M. Gelfand and B. M. Levitan, On the Determination of a differential equation from its spectral function, Izv. Akad. Nauk SSSR, Ser. Math., 15, 309-360 (1951).

[11] M. G. Gasymov and B. M. Levitan, On the Sturm-Liouville differential operators with discrete spectra, Amer. Math. Soc. Transl. Ser., 68, 2 (1968).

[12] V. A. Marchenko, Concerning the theory of a differential operator of the second order, Dokl. Akad. Nauk.(N.S.), 72, 457-460 (1950).

[13] N. Levinson, The inverse Sturm-Liouville problem, Mat. Tidsskr. B, 25-30 (1949).

[14] E. L. Isaacson and E. Trubowitz, The inverse SturmLiouville problem. I, Comm. Pure Appl. Math., 36, 767-783 (1983).
[15] V. A. Yurko, Inverse spectral problems for linear differential operators and their applications, Gordon and Breach, New York, (2000).

[16] A. McNabb, R. Anderssen and E. Lapwood, Asymptotic behaviour of the eigenvalues of a Sturm-Liouville sytstem with discontinuous coefficients, J. Math. Anal. Appl., 54, 741-751 (1976).

[17] C. F. Coleman and J. R. Mclaughlin, Solution of inverse spectral problems for an impedance with integrable derivative, I, II, Commun. Pure Appl. Math., 46, 145-184, 185-212 (1993)

[18] G. Freiling and V. A. Yurko, Inverse problems for differential equations with turning points, Inverse Probl., 13, 1247-1263 (1997).

[19] R. Carlson, An inverse spectral problem for SturmLiouville operators with discontinuous coefficients, Proc. Am. Math. Soc., 120, 475-484 (1994).

[20] L. Andersson, Inverse eigenvalue problems with discontinuous coefficients, Inverse Probl., 4, 353-397 (1988).

[21] O. H. Hald, Discontiuous inverse eigenvalue problems, Comm. Pure Appl. Math., 37, 539-577 (1984).

[22] G. Freiling and V. A. Yurko, Inverse Sturm-Liouville problems and their applications, NOVA Science Publishers, New York, (2001).

[23] V. A. Yurko, Integral transforms connected with discontinuous boundary value problems, Integral Transforms Spec. Funct., 10, 141-164 (2000).

[24] R. Kh. Amirov, On Sturm-Liouville operators with discontinuity conditions inside an interval, J. Math. Anal. Appl., 317, 163-176 (2006).

[25] M. G. Gasymov and G. Sh. Guseinov, Determining of the diffusion operator from spectral data, Dokl. Akad. Nauk Azerb. SSR., 37, 19-23 (1981).

[26] G. Sh. Guselnov, On the spectral analysis of a quadratic pencil of Sturm-Liouville operators, Doklady Akademii Nauk SSSR, 285, 1292-1296 (1985), English translation, Soviet Mathematics Doklady, 32, 859-862 (1985).

[27] G. Sh. Guseřnov, Inverse spectral problems for a quadratic pencil of Sturm-Liouville operators on a finite interval, in Spectral Theory of Operators and Its Applications, Èlm, Baku, Azerbaijan, 51-101 (1986).

[28] H. Koyunbakan, Etibar S. Panakhov, Half-inverse problem for diffusion operators on the finite interval, J. Math. Anal. Appl., 326, 1024-1030 (2007).

[29] V. A. Yurko, An inverse problem for pencils of differential operators, Matem. Sbornik, 191, 137-160 (2000); English transl., Sbornik: Mathematics, 191, 561-1586 (2000).

[30] S. A. Buterin and V. A. Yurko, Inverse spectral problem for pencils of differential operators on a finite interval, Vestnik Bashkir. Univ., 4, 8-12 (2006).

[31] Chuan-Fu Yang and Yong-Xia Guo, Determination of a differential pencil from interior spectral data, J. Math. Anal. Appl., 375, 284-293 (2011).

[32] R. Kh. Amirov and A. A. Nabiev, Inverse problems for the quadratic pencil of the Sturm-Liouville Equations with impulse, Abstract and Applied Analysis, Article ID 361989, (2013).

[33] I. M. Nabiev, Inverse periodic problem for a diffusion operator, Transactions of Academy of Sciences of Azerbaijan, 23, 125-130 (2003). 
[34] I. M. Nabiev, The inverse spectral problem for the diffusion operator on an interval, Matematicheskaya Fizika, Analiz, Geometriya, 11, 302-313 (2004)

[35] A. Sh. Shukurov, The inverse problem for a diffusion operator, Proceeding of IMM of NAS of Azerbaijan, 105-110 (2012).

[36] M. Sat, Etibar S. Panakhov, Spectral problem for diffusion operator, Applicable Analysis, (2013),

[37] Y. P. Wang, A uniqueness theorem for diffusion operators on the finite interval, Acta Mathematica Scienta, 33A, 333-339 (2013).

[38] H. Weyl , Über gewohnliche differentialgleichungen mit singularitäten und die zugehörigen entwicklungen willkürlicher funktionen, Math. Ann., 68, 220-269 (1910) .

[39] B. F. Jdanovich, Formulae for the zeros of Drichlet polynomials and quasi-polynomials, Doklady Akademii Nauk SSSR, 135, 1046-1049 (1960).

[40] B. Ya Levin, Entire Functions. MGV, Moscow, Russia, (1971).

[41] R. Bellman and K. L. Kuk, Difference-differential equations. M., "Mir", (1967), (Russian).

[42] V. A. Marchenko, Sturm-Liouville problems and their applications, Naukova Dumkas, Kiev, (1977). English Trans. Birkhauser, (1986).

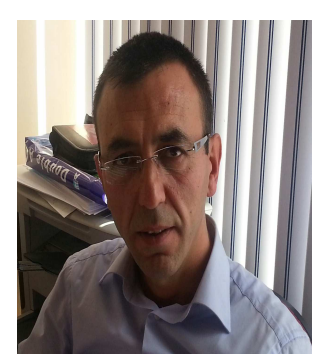

Yaşar

Çakmak

received the $\mathrm{PhD}$ degree

in Mathematics Science

at Cumhuriyet University

of Sivas. His research

interests are in the areas

of applied mathematics

and mathematical physics

including the mathematical methods of spectral analysis.

He has published research articles related with direct and inverse spectral problems for differential operators.

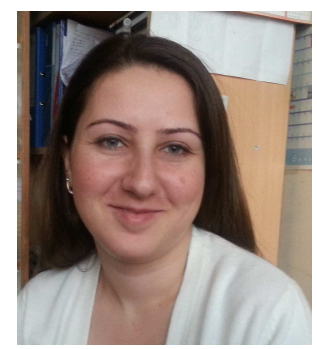
the $\mathrm{PhD}$ degree in Mathematics Science at Cumhuriyet University of Sivas in 2013. She still works at the Cumhuriyet University, Faculty of Education, Department of Secondary School Science and Mathematics Education 